\title{
Radiolysis of ammonia-containing ices by heavy cosmic rays inside dense molecular clouds
}

\author{
Sergio Pilling ${ }^{1,2}$, Eduardo Seperuelo Duarte ${ }^{1,3,4}$, Enio F. da Silveira ${ }^{1}$, \\ Emmanuel Balanzat ${ }^{3}$, Hermann Rothard ${ }^{3}$, Alicja Domaracka ${ }^{3}$ and \\ Philippe Boduch ${ }^{3}$ \\ ${ }^{1}$ Pontifícia Universidade Católica do Rio de Janeiro (PUC-Rio), 22453-900, \\ Rio de Janeiro, RJ, Brazil. \\ ${ }^{2}$ Instituto de Pesquisa e Desenvolvimento (IP\&D), Universidade do Vale do Paraíba \\ (UNIVAP), 12244-000, São José dos Campos, SP, Brazil \\ ${ }^{3}$ Centre de recherche sur les ions, les matériaux et la photonique (CEA /CNRS /ENSICAEN \\ /Université de Caen-Basse Normandie), CIMAP-CIRIL-GANIL, Caen, France. \\ ${ }^{4}$ Grupo de Física e Astronomia, CEFET/Química de Nilópolis, 12653-060, Nilópolis, Brazil.
}

\begin{abstract}
We present experimental studies on the interaction of heavy, highly charged and energetic ions ( $\left.46 \mathrm{MeV}^{58} \mathrm{Ni}^{13+}\right)$ with interstellar ammonia-containing $\left(\mathrm{H}_{2} \mathrm{O}: \mathrm{NH}_{3}: \mathrm{CO}\right)$ ice analog in an attempt to simulate the physical chemistry induced by heavy ion cosmic rays inside dense astrophysical environments. The measurements were performed at the heavy ion accelerator GANIL in Caen, France. In-situ analysis have been performed by a Fourier transform infrared spectrometer. The averaged values for the dissociation cross section of water, ammonia and carbon monoxide are determined and the estimated half life for the studied species inside dense molecular clouds is $2-3 \times 10^{6}$ years. The IR spectra of organic residue produced by the radiolysis have revealed, at room temperature, five bands that are tentatively assigned to vibration modes of the zwitterionic glycine $\left(\mathrm{NH}_{3}^{+} \mathrm{CH}_{2} \mathrm{COO}^{-}\right)$.
\end{abstract}

Keywords. astrochemistry, astrobiology, molecular processes, molecular data, methods: laboratory, ISM: clouds, cosmic rays

\section{Introduction}

The observation of molecules in the gas phase deeply inside these cold and dense regions, where the gas sticking efficiency on grains is close to unity, suggests that they are indeed energetically active regions (e.g. (Ehrenfreund \& Charnley (2000)). In these highly obscured regions, cosmic rays are the main energy source for chemical reactions. Although the flux of heavy ions (e.g. Fe, $\mathrm{Ni}, \mathrm{Si}, \mathrm{Mg}, \ldots$ ) is about 3-4 orders of magnitude lower than that of protons (Roberts et al. (2007)), their effects play an important role on interstellar grains since they can deposit about 100 times more energy than the light ions (protons and alpha particles) inside the grains. The amount of species released per impact to gas phase due to heavy ions could be 4-5 orders of magnitude higher than those for protons (Seperuelo Duarte et al. (2009)).

\section{Experimental methodology and results}

In an attempt to simulate the physical chemistry induced by heavy ion cosmic rays inside dense astrophysical environments we have performed radiolysis experiments on a $\mathrm{H}_{2} \mathrm{O}: \mathrm{NH}_{3}: \mathrm{CO}$ (1:0.6:0.4) $13 \mathrm{~K}$ ice mixture at the heavy ion accelerator GANIL (Grand 
Accelerateur National d'Ions Lourds) in Caen, France. In-situ Fourier transformed infrared (FTIR) spectra were recorded at different fluences, up to $2 \times 10^{13} \mathrm{Ni}_{\text {ions } \mathrm{cm}^{-2}}$. Further experimental information is given elsewhere(Seperuelo Duarte et al. (2009); Pilling et al. (2009)).

The dissociation cross sections: $\sigma_{H 2 O} \sim 2 \times 10^{-13}, \sigma_{C O}=1.4 \times 10^{-13}$ and $\sigma_{N H 3}=$ $1.9 \times 10^{-13} \mathrm{~cm}^{2}$ were determined from the evolution of the molecular column density as a function of ion fluence. Figure 1a presents a comparison between the IR spectra of interstellar ices around the embedded protostar W33A obtained by Infrared Space Observatory (ISO) with the irradiated water-ammonia-CO ice at a fluence $2 \times 10^{13} \mathrm{Ni}$ ions $\mathrm{cm}^{-2}$. The bands observed from the radiolysis reproduces very well the $\mathrm{OCN}^{-}(2165$ $\left.\mathrm{cm}^{-1}\right)$ and CO $\left(2139 \mathrm{~cm}^{-1}\right)$ IR features observed in the W33A spectrum. Other spectral features like the broad IR peaks observed at 1650 and $1450 \mathrm{~cm}^{-1}$ are also similar to the astronomical source. The IR analysis of organic residue at $300 \mathrm{~K}$ suggests the presence of containing $\mathrm{CN}$ bearing molecules, amides, esters and possibly zwitterionic glycine $\left(\mathrm{NH}_{3}^{+} \mathrm{CH}_{2} \mathrm{COO}^{-}\right)$(Figure $1 \mathrm{~b}$ ). The complete description of the results can be found elsewhere Pilling et al. (2009).
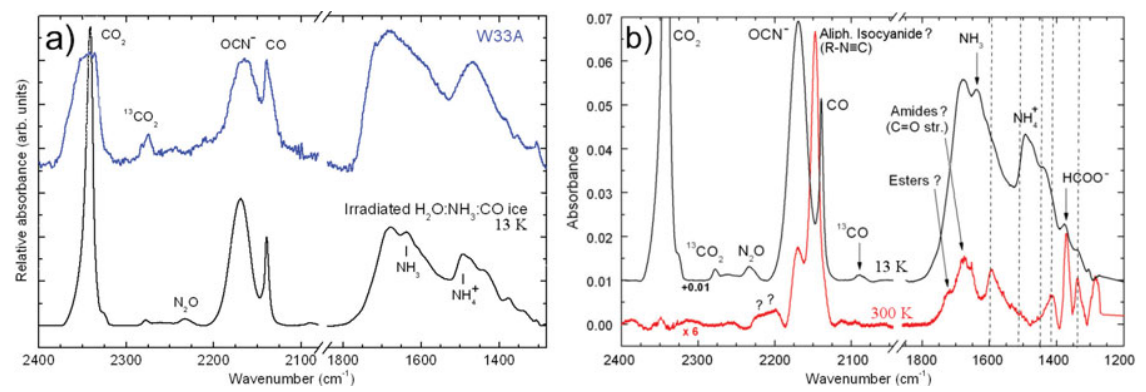

Figure 1. a) Comparison between the IR spectra of protostar W33A obtained by Infrared Space Observatory (ISO) with the irradiated $\mathrm{H}_{2} \mathrm{O}: \mathrm{NH}_{3}$ : $\mathrm{CO}(1: 0.6: 0.4) 13 \mathrm{~K}$ ice at a fluence $2 \times 10^{13} \mathrm{Ni}$ ions $\mathrm{cm}^{-2}$. (b) Comparison between the irradiated ice and the $300 \mathrm{~K}$ residue. Vertical dashed lines indicate the frequencies of some vibration modes of zwitterionic glycine $\left(\mathrm{NH}_{3}^{+} \mathrm{CH}_{2} \mathrm{COO}^{-}\right)$. Details can be found elsewhere (Pilling et al. (2009))

\section{Conclusions}

Experimental study on the interaction of energetic Ni ions on ammonia-containing interstellar ice analog have been performed to simulate the physical chemistry induced by cosmic rays inside dense regions of interstellar medium. Dissociation cross section have been determined allowing an estimative for half life of the studied species of about $2-3 \times 10^{6}$ years in real astrophysical real situation. This value is in a good agreement with the half lives of typical dense molecular environments in the interstellar medium. Although they represent only a small fraction $(\sim 1 \%)$ of the cosmic rays flux, some effects (e.g. molecular sputtering and ice compaction) promoted by heavy ions on interstellar ice grains are more intense than those promoted by protons.

\section{References}

Ehrenfreund, P. \& Charnley, S. B. 2000, ARAA, 38, 427

Roberts, J. F., Rawlings, J. M. C., Viti, S., \& Williams, D. A. 2007, MNRAS, 382, 733

Seperuelo Duarte, E., Boduch, P., Rothard, H., Been, T., Dartois, E., et al. 2009, A\&SA, 502, 599

Pilling, S., Seperuelo Duarte, E., da Silveira, E. F., Balanzat, E., et al. 2009 A 6 A, Aceppted 


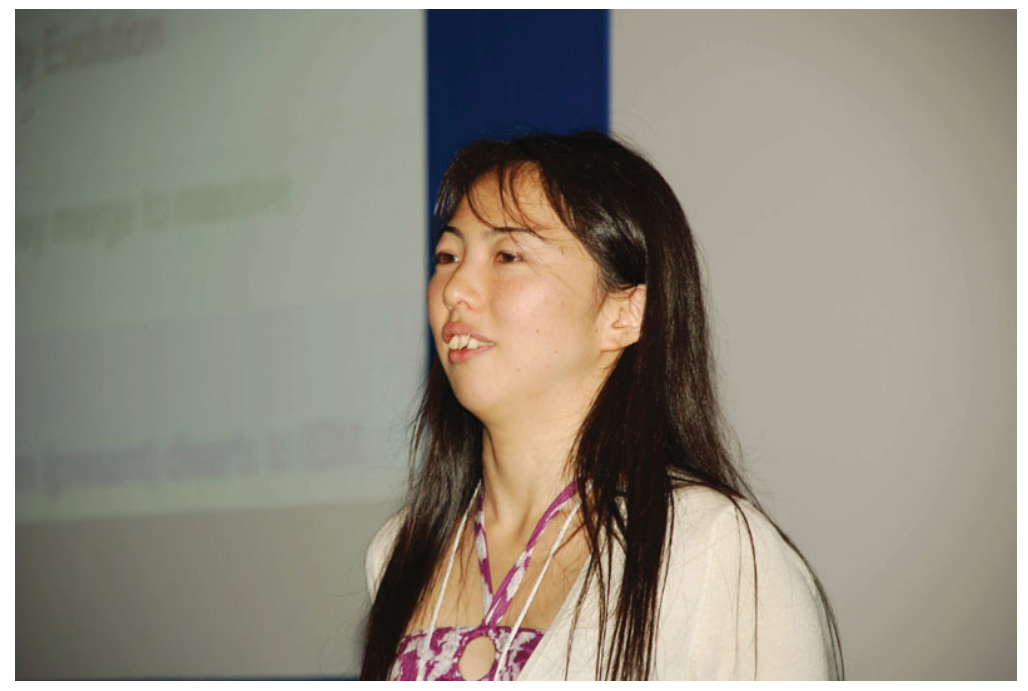

Chiaki Kobayashi during her talk.

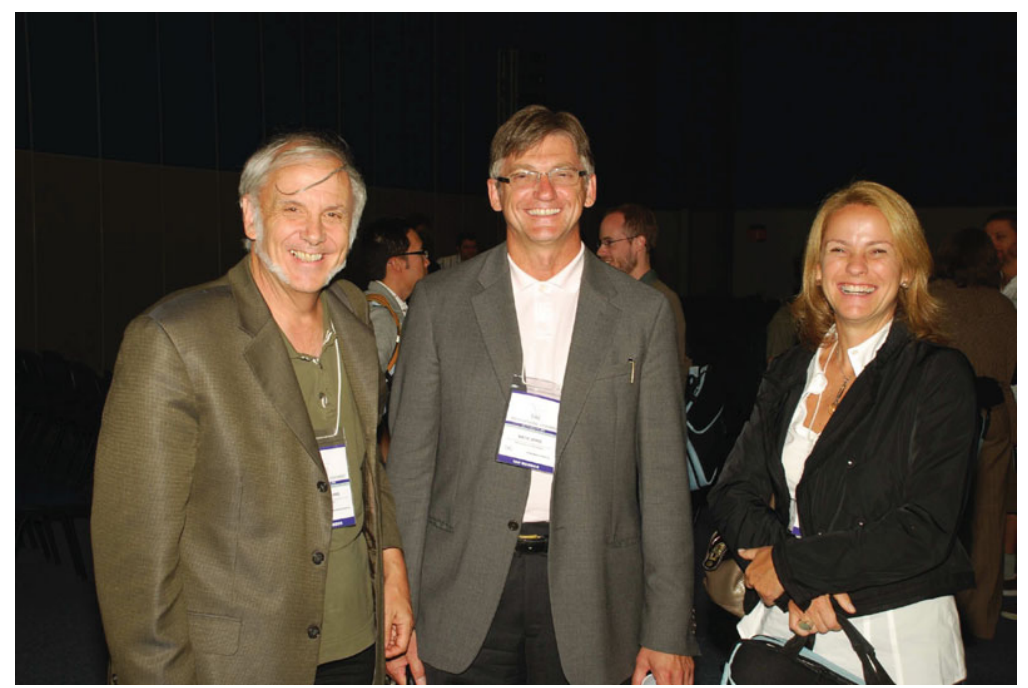

Chris Sneden, Verne Smith and Katia Cunha. 